\title{
Droplet-Based Microgels: Attractive Materials for Drug Delivery Systems
}

\section{ISSN: 2576-8840}

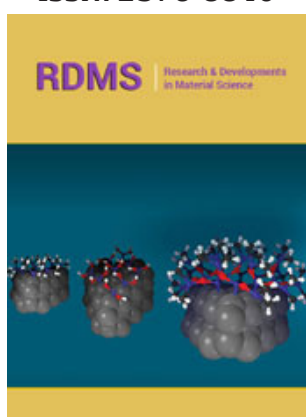

*Corresponding author: Sammer-ul Hassan, Mechanical Engineering, Faculty of Engineering and Physical Sciences, UK

Submission: 制 August 12, 2019

Published: 侮 August 20, 2019

Volume 11 - Issue 3

How to cite this article: Sammer-ul $\mathrm{H}$ Xunli Z. Droplet-Based Microgels: Attractive Materials for Drug Delivery Systems. Res Dev Material Sci.11(3).RDMS.000763.2019. DOI: 10.31031/RDMS.2019.11.000763

Copyright@ Sammer-ul Hassan, This article is distributed under the terms of the Creative Commons Attribution 4.0 International License, which permits unrestricted use and redistribution provided that the original author and source are credited.
Sammer-ul Hassan ${ }^{1,2 *}$ and Xunli Zhang ${ }^{1,2}$

${ }^{1}$ Mechanical Engineering, Faculty of Engineering and Physical Sciences, University of Southampton, Southampton, S017 1BJ, UK

${ }^{2}$ Institute for Life Sciences, University of Southampton, Southampton, S017 1BJ, UK

\begin{abstract}
Microgels are materials that have the properties to swell and shrink when exposed to external stimuli, i.e. temperature and $\mathrm{pH}$. Therefore, they are instrumental in the field of drug delivery, but their production is complicated. Different strategies have been used to produce microgels from which emulsion polymerization and microfluidic techniques are commonly used. Lab-on-a-chip devices are handy and alternative tools to produce microgels because they provide control on the size, shape and reproducible production of microgels. This review will highlight the ways how to initiate polymerization inside droplets and convert them into microgels using lab-on-a-chip devices.
\end{abstract}

Keywords: Microgels; Microfluidics; Miniaturisation; Droplet microfluidics; Polymers

\section{Mini Review}

A microgel as defined by IUPAC is "particle of gel of any shape with an equivalent diameter of approximately 0.1 to $100 \mu \mathrm{m}$ ". Baker's group was one of the first that studied microgels, and "micro" refers to the size and "gel" refers to the structure of the particle [1]. Thus, microgels are cross-linked polymer chains and are similar to macrogels, but they are in the micro-scale. Because of their micro-scale size, microgels have advantages over macrogels, and some of them are mentioned here. i) Microgel suspensions are free-flowing liquids which depend upon volume fraction of swollen particles and cross-linking properties. ii) Microgels respond to changing environment very rapidly, much faster than macrogels, because they have a high surface to volume ratio which transports mass to-and-from the microgels. iii) Exotic microgel morphologies can be used to fine-tune properties. iv) Microgels are considered as colloidal dispersions, and several colloidal science techniques including dynamic light scattering and small range light can provide structural information for microgels. On the other hand, these techniques cannot be used in the case of macrogels. v) Microgels can be assembled into useful larger objects such as 2D assemblies of the air-water and oil-water interfaces [2]. Examples of the 3D structure are colloidal crystals, environmentally sensitive optical properties, and layer-by-layer assemblies. These kinds of assemblies are not feasible when using macrogels.

Microgels consist of a soft domain and can change shape and size in response to mechanical stress, temperature or chemical environment (like $\mathrm{pH}$ and ionic strength). The mechanical behaviour and properties of the microgel can quickly be tailed during synthesis. Furthermore, microgels can also be functionalized with special chemical groups. These microgels are attractive materials for many applications like drug delivery. For example, a drug can be encapsulated within the microgel and then released when changing the $\mathrm{pH}$ or the temperature [3]. There are two primary methodologies to synthesize microgels, i.e. emulsion polymerization and droplet-based microfluidics. These two methodologies are discussed in this review.

Emulsion polymerization is dispersions involving monomers of limited water-solubility and an emulsifying agent (synthetic detergent), the detergent forms small micelles, smaller than droplets by chemical aggregation in suspension. Micelles that are formed contain small amounts of monomers in water without surfactant. The emulsion polymerization is initiated using water-soluble initiators, therefore, must not be soluble in the monomer but soluble in aqueous media and cross-linker, forming a free radical or growing chain in solution. When passed through micelle and diffused into them, causing bulk polymers in 
the stabilized droplet, therefore, viscosity increases resulting in a dispersed polymer. Microgel synthesis must account for controlling particle size distribution, the colloidal stability, and distribution of specific functional groups (cross-linker, charged groups) are reactive for further chemical derivatization. Thus, Ionic surfactants are often used as dispersion stabilizers with $1-5 \mathrm{wt} \%$ levels to the monomer. Emulsion polymerization is limited to form droplets of ten to hundreds of nanometers. The narrow size distribution from a submicron level, membrane emulsification or shear cell, are typically used for the templating for emulsion droplets to be produced as a bulk method. This reduced control over individual drop formation results in polydisperse ensembles of droplets. This is detrimental if product particles shall serve for encapsulation or control release purpose, which requires a degree of loading; therefore, size and volume needs to be uniform. The microfluidic approach is a great advantage to overcome bulk emulsification limitations [4].

Over the last decade, there has been an increase in the development of tools for fluid flow of highly monodispersed droplets in nano-micro diameter range along with their particle shape, and this is an ideal innovation to perform lab operations using fraction and volume of reagents in less time. The multidisciplinary technology involves applications that vary from drug delivery to the point-of-care diagnostics to organic synthesis and micro reactions. Controlling flow rate, surface tension and weight of the drop, these constant parameters of the droplet-based manipulation of discrete drop formation in a microdevice can be demonstrated using a water faucet as an example, thus resulting in the narrow size distribution of drops dripping from the faucet can be displayed [5].

There are two ideal techniques developed to fabricate microfluidic chips for droplet generation, i.e. glass microchips and soft lithography. Glass microcapillaries are fabricated to form a coaxial alignment with inner and outer dimensions which can produce more sophisticated droplets from the flow, e.g. spherical dimensions, double emulsions and same shape. Advantage of this morphology is highly resistant to chemicals and wettability is controlled by quick surface reaction with the appropriate modifier. Glass chips are widely used for fabrication of microfluidic devices because the chip is transparent allowing for performing light-based experiments. However, the glass microchip manufacturing process is relatively complicated as compared to PDMS devices. It is also hydrophilic and facilitates on-chip oil droplet-based experiments. A typical chip is made of two glass layers that are fused. The bottom layer is the bulk with the chip architecture designed on its surface. The top part is a thin layer of glass that acts as a cover. When the two parts are connected, a piece of glass is created with the ducts and designed features being hollow elements inside of the chips. Chips made by Soft lithography are fabricated with the principle of mould microfluidic device by drawing to scale of the channels of the device on transparency and transferring the image to a photoreactive substrate. This allows small channels to be made with the level of precision and flexibility over design channel geometry [6].
The main advantage of preparing microgels in microfluidic devices is the fact that the droplets formed by the device have the same size. Thus, the size distribution of the microgels is very narrow, and it can also easily be tailored by changing the settings of the microfluidics device. In addition to single emulsion microchips, the multiple emulsion microchips can also be used to generate microgels inside microgels. This report aims to summarise and review studies where they have used single emulsion droplet-based microfluidics to synthesize microgels. There are two techniques mainly used to generate droplets, i.e. flow-focusing and T-junction. The droplets can be manipulated inside the microfluidic channels and can be directed to chambers where they can be heated and exposed to UV light to initiate polymerization inside droplets $[5,6]$.

There are two main methods used to convert droplets into microgels depending upon the use of the material [7]. Firstly, a small monomer, cross-linker and initiator are mixed within the droplet. The initiator is activated with the help of UV light and heat, which initiates the polymerization of the monomer and the crosslinker. The monomer forms the polymer chains, and the crosslinker links the chains together to form the microgel. Secondly, a larger monomer(s) or cross-linker(s) that are sometimes also called macromonomer or macro cross-linker are mixed with the initiator. A more generic term to use for both type of molecules is pre-polymers. Then by application of UV or heat, the initiator is activated, which initiates the polymerization, and the microgel is formed. For example, Seiffert et al. [8] generated droplets of polymerised materials using a flow-focusing device and directed them to a channel where they were exposed to intense UV light. The concept of polymerization inside miniaturised systems has been studied intensively, and different strategies have been used to generate droplets of polymerised materials in the form of microgels $[7,8]$. Additional properties can be imparted into the microgels by embedding functional materials into microgels. Thermo-responsive microgels can be added with nanoparticles, quantum dots etc., but these functional materials do not affect the thermo-responsive behaviour of the microgels. Such as enzymes were loaded with microgels, and there were no responsive problems found with microgels [9]. On the other hand, the voids were added to the microgel, and these were found to be worked efficiently [10].

Despite having all the persuasive techniques, the polymerisation inside droplets is difficult with some polymers. Pre-polymer can be more useful, which can provide microgels in one step. The droplets can be made from macromolecular precursors, and then these droplets can be solidified using UV light. There are a couple of successful approaches which have produced microgels from pre-polymers [11-13]. Pre-polymers are very useful as they can be prepared separately, and functional materials can also be introduced into the precursor solutions; therefore, they can provide $100 \%$ efficiency in the microfluidic systems. Future work must relate to the formation of smart microgels or stimuli response microgels which have the properties to shrink and swell. These smart microgels must allow the drugs delivery at targeted 
tissues with the change in external stimuli like temperature. The release of drugs could be very useful to treat specific diseases such as tumours. Therefore, microgels have the potential to retain functional materials inside its polymeric network and can have very vast applications in the field of drug delivery.

\section{Acknowledgement}

We thank the funding from the Economic and Social Research Council UK (ES/S000208/1).

\section{References}

1. Graham NB, Cameron A (2007) Nanogels and microgels: The new polymeric materials playground. Pure Appl Chem 70(6): 1271-1275.

2. Fernandez-Nieves A, Wyss HW, Mattsson J, Weitz DA (2011) Microgel suspensions: fundamentals and application. Wiley-VCH Verlag $\mathrm{GmbH} \&$ Co. KGaA.

3. Karg M, Hellweg T (2009) New 'smart' poly (NIPAM) microgels and nanoparticle microgel hybrids: Properties and advances in characterization. Current Opinion in Colloid and Interface Science 14(6): 438-450.

4. M. Poliskie (2011) Introduction to Polymers. Solar Module Packaging.

5. The SY, Lin R, Hung LH, Lee AP (2008) Droplet microfluidics. Lab on a Chip.
6. Huebner A, Sharma S, Srisa-Art M, Hollfelder F, Edel JB, et al. (2008) Microdroplets: a sea of applications? Lab Chip 8(8): 1244-1254.

7. Krause E, Borner HG (2004) Macromolecular rapid communications. Macromol Rapid Commun 40(16).

8. Seiffert S, Weitz DA (2010) Microfluidic fabrication of smart microgels from macromolecular precursors. Polymer 51(25): 5883-5889.

9. Jeong WJ (2005) Continuous fabrication of biocatalyst immobilized microparticles using photopolymerization and immiscible liquids in microfluidic systems. Langmuir 21(9): 3738-3741.

10. Chu LY, Kim JW, Shah RK, Weitz DA (2007) Monodisperse thermoresponsive microgels with tunable volume-phase transition kinetics. Adv Funct Mater 17(17): 3499-3504.

11. Xu S (2005) Generation of monodisperse particles by using microfluidics: Control over size, shape, and composition. Angew Chemie Int Ed 44(5): 724-728.

12. Zhang H, Tumarkin E, Sullan RMA, Walker GC, Kumacheva E (2007) Exploring microfluidic routes to microgels of biological polymers. Macromol. Rapid Commun 28(5): 527-538.

13. Tumarkin E, Kumacheva E (2009) Microfluidic generation of microgels from synthetic and natural polymers. Chemical Society Reviews 38(8): 2161-2168. 